\title{
Potential applications for biguanides in oncology
}

\author{
Michael Pollak
}

Department of Oncology, McGill University, Montreal, Quebec, Canada.

\begin{abstract}
Metformin is widely prescribed for the treatment of type II diabetes. Recently, it has been proposed that this compound or related biguanides may have antineoplastic activity. Biguanides may exploit specific metabolic vulnerabilities of transformed cells by acting on them directly, or may act by indirect mechanisms that involve alterations of the host environment. Preclinical data suggest that drug exposure levels are a key determinant of proposed direct actions. With respect to indirect mechanisms, it will be important to determine whether recently demonstrated metformin-induced changes in levels of candidate systemic mediators such as insulin or inflammatory cytokines are of sufficient magnitude to achieve therapeutic benefit. Results of the first generation of clinical trials now in progress are eagerly anticipated. Ongoing investigations may justify a second generation of trials that explore pharmacokinetic optimization, rational drug combinations, synthetic lethality strategies, novel biguanides, and the use of predictive biomarkers.
\end{abstract}

\section{Introduction}

There is renewed interest in treatments that modulate metabolism to limit neoplastic growth. Metabolic therapies have been studied in the past, in part because the classic studies of Otto Warburg (1) raised hopes that targeting glycolysis would be of therapeutic benefit. While agents such as 2-deoxyglucose have not proven clinically useful, targeting energy metabolism remains a key research focus. There is evidence that the biguanide family of compounds induces energetic stress by inhibition of oxidative phosphorylation (2-7), but additional mechanisms have not been excluded $(8,9)$. A long history of research supports the idea that biguanides perturb cellular and whole-organism energy metabolism; the challenge is to determine whether this can be exploited for cancer treatment or prevention (see Milestones in biguanide research related to neoplasia).

A strict interpretation of Warburg's original hypothesis would suggest that the direct action of biguanides as inhibitors of oxidative phosphorylation is not attractive for cancer treatment, as mitochondrial dysfunction was believed to be a key component of cancer pathophysiology, leading to excess glycolysis. However, more recent work suggests that cancer cells remain reliant on mitochondria for ATP production and other metabolic functions, and that there are contexts in which inhibition of oxidative phosphorylation may be therapeutically useful.

Besides diabetes, established indications for metformin include polycytic ovary disease (10) and obesity induced by antipsychotic medications (11). Importantly, the actions of metformin in reducing glucose levels and correcting hyperinsulinemia vary with physiologic context: while metformin can dramatically lower the high insulin and glucose levels found in type II diabetes, it has relatively modest effects when administered to subjects with normal levels at baseline.

Pharmacoepidemiologic research provided early clues of possible relevance of metformin to neoplastic disease. An early report (12) and later studies (reviewed in refs. $2,13,14$ ) provided retrospective evidence that diabetics treated with metformin have unexpectedly low incidence of cancer and/or reduced cancer mortality. Many of these findings have recently been criticized on methodologic grounds $(15,16)$. Some reported protective effects are of a magnitude unprecedented among cancer prevention studies, such

Conflict of interest: The author has declared that no conflict of interest exists. Citation for this article: J Clin Invest. 2013;123(9):3693-3700. doi:10.1172/JCI67232. as examples of $80 \%$ risk reduction for hepatocellular cancer (17, 18). Results of this kind require careful assessment in terms of methods and validity: if confirmed, these findings could lead to dramatic clinical benefits, but if unfounded could lead to inappropriate justification for randomized clinical trials. In the case of breast cancer, many trials of metformin are ongoing. When these trials were planned, available pharmacoepidemiologic evidence supported the rationale (for example, ref. 19), but more recent results are less persuasive (for example, ref. 20).

The early pharmacoepidemiology results motivated several groups to carry out laboratory studies. To the surprise of some observers, antineoplastic activity was indeed observed in early in vitro (21) and in vivo (22) laboratory models and in many subsequent studies (reviewed in ref. 2). While most of the laboratory evidence for antineoplastic activity of metformin was observed at higher exposure levels than achieved during diabetes treatment, these findings contributed to the rationale for the large number of ongoing clinical trials of metformin for various indications in oncology.

Mechanisms of action of potential relevance to oncology Although many studies have described metformin-induced physiologic changes that correlate with antiproliferative activity in model systems, formal evidence that particular alterations are necessary and sufficient for this activity is often lacking, and much research is ongoing.

The primary target. Despite extensive research, the precise mechanism of biguanides at the molecular level remains poorly understood; however, available data suggest that inhibition of oxidative phosphorylation due to reduced function of respiratory complex I underlies cellular and whole organism actions $(3-7,23)$, and recent results show that metformin inhibits mitochondrial complex I by binding reversibly to the functionally critical hydrophilichydrophobic domain interface (J. Hirst, personal communication). It is important to recognize that pharmacokinetic factors operating at both the cellular and whole organism levels result in heterogeneity between tissues in exposure to biguanides (24, 25). For example, following oral administration, liver is exposed to relatively high levels due to high concentrations in the portal circulation. Additionally, metformin and other less lipophilic biguanides are transported into cells by membrane transport proteins such as organic cation transporter 1 (OCT1), so bioactivity varies between cell types according to expression level of transport 


\section{Milestones in biguanide research related to neoplasia}

Time
Medieval times
1920 s
1990 s
2000
2005
2006
2008

2011

2012

2014

Future

\section{Event}

Use of guanadine-rich herbs for diabetes treatment

Early clinical investigations of biguanides for diabetes treatment

Metformin becomes one of the most widely prescribed drugs in the world

Research begins to clarify metformin mechanisms of action relevant to diabetes treatment

Pharmacoepidemiologic clues are discovered for antineoplastic actions of metformin

Laboratory models are developed to mimic the antineoplastic effects of metformin

Clinical trials planned for antidiabetic doses of metformin in cancer patients, but without specific molecular selection criteria

Research intensifies regarding fundamental molecular pharmacology of metformin action

Research begins into cancers sensitive to energetic stress and strategies to achieve synthetic lethality with biguanides

Research begins into optimization of biguanide pharmacokinetics

Concern arises about the validity of early pharmacoepidemiology studies

Initial results are expected from randomized clinical trials investigating the efficacy of metformin in treatment of advanced cancers

Results expected from studies of novel biguanide or new dosing regimes of metformin

Results expected from studies of rational combinations of biguanide drugs with other anticancer therapies

Results expected from studies of subsets of tumors selected for sensitivity to biguanides

Results expected from studies of organ sites and cancer predisposition syndromes in which metformin may be a preventative therapeutic agent molecules (26). Within cells, metformin is non-uniformly distributed, with high concentrations accumulating in the mitochondria, driven by membrane potential.

Examples of systemic effects. Systemic effects result from the subset of local effects that influence whole organism homeostasis (Figure 1). The most obvious example concerns the effect of biguanides on gluconeogenesis, which accounts for much of their activity in treatment of type II diabetes. The relatively high level of metformin in the liver results in reduced complex I activity in hepatocytes, causing energetic stress. This stress, in turn, by LKB1/AMPKdependent (27) and/or -independent (28) mechanisms, inhibits gluconeogenesis, which has systemic effects including reduced hepatic glucose output, reduced serum glucose, and a reduction in hyperinsulinemia (if present at baseline). The extent of reduction of hyperglycemia and hyperinsulinemia is greater in the setting of high baseline levels, and the effect is more pronounced on postprandial levels than fasting levels (M.-J. Blouin, personal communication). Elevated serum levels of insulin or c-peptide (which vary with insulin secretion) are associated with more aggressive behavior and/or increased risk of breast cancer $(29,30)$, colon cancer $(31,32)$, and prostate cancer (33) and with rapid tumor growth in certain model systems (for example, refs. 34-36). Thus, in situations in which metformin leads to substantial reduction in insulin levels, this may inhibit the growth of the subset of tumors that are insulin responsive $(2,37,38)$.

Other systemic effects of biguanides may arise from effects on effector cells in the immune system (39-41) that may influence chronic inflammatory states that favor neoplasia. Clinical research in this area is revealing effects of metformin not previously characterized in detail, including effects on inflammatory mediators and resistin $(42,43)$. Additional systemic effects of metformin related to reduction in glucagon signaling, attributable to the effects of the drug on ATP production in glucagonresponsive cells, may also be important (44). Early results further suggest a systemic effect of metformin on adiponectin levels (45). Fascinating evidence indicates that the effect of metformin as a life-extending agent in C. elegans is attributable to a systemic effect resulting from a direct action on folate metabolism in gut bacteria (46), but the potential clinical relevance of this work remains an active research topic. An effect of biguanides on folate metabolism in human cells has also been reported (47).

Examples of direct effects on neoplastic cells. Energetic stress due to biguanide-induced decrease in oxidative phosphorylation results in a variety of consequences, which vary with physiologic context and the characteristics of targeted cells. While most neoplastic cells exhibit increased glycolysis, as originally described by Warburg, mitochondrial ATP production is not dispensable. Even when glycolytic flux is high, oxidative phosphorylation contributes substantially to ATP production, and also is critical for other metabolic functions, including maintenance of redox balance to permit function of the TCA cycle (48-50).

Direct actions of biguanides on transformed cells or cells at risk for transformation may be a consequence of homeostatic responses to biguanide-induced energetic stress or may be attributable to ATP depletion itself (ref. 2 and Figure 2). The LKB1/AMPK system acts as a sensor of ATP level, and downstream pathways act to minimize energy utilization and maximize ATP generation $(51,52)$. Early reports (for example, ref. 21) showed that metformin-induced inhibition of oxidative phosphorylation leads to AMPK activation and to AMPK-dependent antiproliferative effects, which are mediated by processes such as AMPK-mediated inhibition of fatty acid synthesis (53) and AMPK-mediated inhibition of mRNA translation (54). 


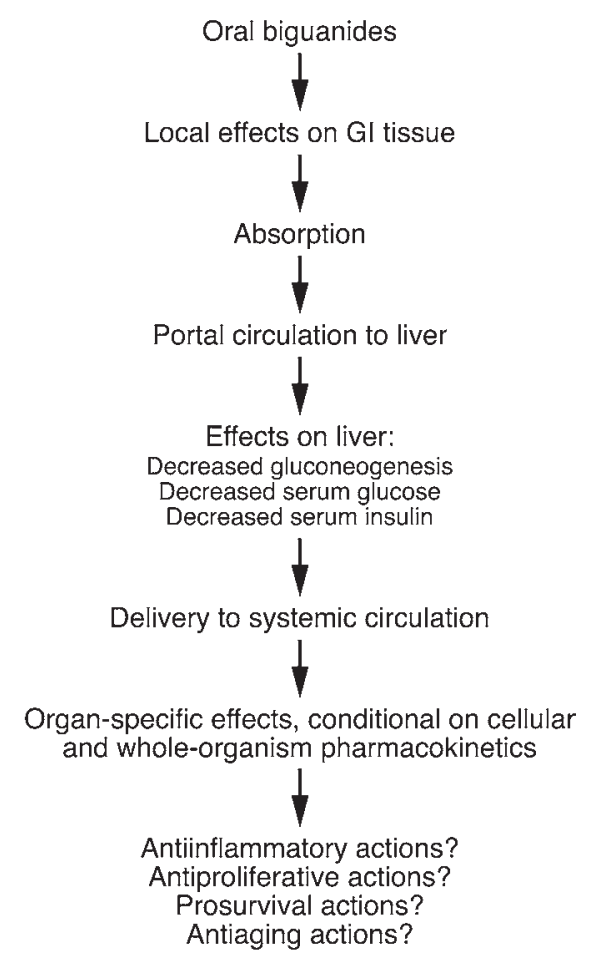

The nature and abundance of carbon source is an important variable to consider $(36,55)$. In the setting of very high glucose concentration (often used in tissue culture media), disabling LKB1/AMPK protects cells from the antiproliferative actions of metformin (21), indicating that this system is a key mediator of the antiproliferative action of the drug under these circumstances. It is apparent that in this context glycolysis is greatly increased and probably provides adequate ATP to compensate for the reduction in oxidative phosphorylation. However, more recent data show that at lower, more physiologic glucose concentrations, disabling LKB1/AMPK actually sensitizes cells to the effects of metformin $(36,56)$. These data are compatible with the notion that if low glucose concentration limits compensatory increases in glycolytic carbon flux when metformin reduces oxidative phosphorylation, the action of LKB1/AMPK in reducing energy consumption becomes critical for cell survival. Thus, in the absence of LKB1/AMPK and in the presence of metformin, energy consumption remains high, while ATP production is decreased, which can result in an energetic crisis and a cytotoxic effect of biguanides. This potential mechanism deserves scrutiny, because loss of LKB1 and/or lack of activation of AMPK is relatively common in neoplasia (57-60).

Recent provocative clinical and experimental data (61) suggest that certain p53 mutations seen in Li-Fraumeni syndrome are associated with increased oxidative phosphorylation. This finding contrasts with prior results from model systems that established that p53 regulates mitochondrial function but implied that p53 loss of function is associated with decreased cellular respiration and increased glycolysis (62). If this result is confirmed and if the upregulation of oxidative phosphorylation is pathophysiologically related to the oncologic sequellae of certain p53 mutations, then reduction of oxidative phosphorylation by biguanides may be of therapeutic value. Indeed, the finding of increased oxidative phosphorylation in the setting of $\mathrm{p} 53$ mutation provides an additional

\section{Figure 1}

Systemic effects of biguanides. Following oral administration, biguanides have local effects on the Gl tract, including the colon, as the luminal concentration can exceed the serum concentration. This elevated concentration may explain observed antiproliferative effects of metformin on colon epithelial cells $(92,93)$. Absorbed drugs are initially routed to the liver via the portal circulation, and this organ is exposed to high concentrations relative to others, not only because it receives portal circulation, but also because hepatocytes express high levels of cell surface transport molecules such as OCT1 required for the import of biguanides such as metformin. In liver, gluconeogenesis is inhibited, leading to declines in circulating glucose and insulin, particularly in the setting of elevated baseline levels. A variety of actions may also occur in other organs, including potentially antiinflammatory actions (40), antiproliferative actions (21), prosurvival actions (83), and antiaging actions (22). However, these effects are dependent on adequate drug levels, and knowledge concerning organ-specific pharmacokinetics of metformin is incomplete.

explanation for prior evidence that tumors with loss of function of p53 are particularly sensitive to metformin. Prior interpretation of this observation (63) was based on the concept that LKB1/AMPKmediated activation of $\mathrm{p} 53$ leading to inhibition of proliferation was an important component of the energy-conserving consequences of AMPK activation, so that p53-defective cells were less likely to successfully conserve energy in the setting of biguanide-induced energetic stress and thus more likely to undergo an energetic crisis.

There is evidence for a differential effect of metformin on stem cells as compared with differential cells (64), which may be relevant to potential oncologic indications (65). The precise basis for this selective toxicity remains an active research topic, and possibilities include the hypothesis that stem cells may be particularly vulnerable to energetic stress.

Finally, evidence (66) suggests that metformin can act directly on target cells in certain situations by inhibiting RAG GTPase protein signaling, resulting in mTOR inhibition, without the involvement of AMPK. The basis for this observation requires further study. There are no data implying that biguanides directly interact with RAG proteins; rather, it is possible that biguanide-induced energetic stress reduces RAG signaling, perhaps by compromising function of v-ATPases (67). Evidence has also been presented for an effect of metformin on mTOR via a primary action on DNA damage-inducible transcript 41 (68).

\section{Rational combinations}

While there may be circumstances in which biguanides have single-agent activity, there is particular interest in exploring opportunities for synthetic lethality. Many combinations have been proposed, but few have been thoroughly evaluated in models. Several proposed biguanide combinations are discussed below.

Inhibitors of glycolysis. One of the earliest suggestions was to combine biguanides with inhibitors of glycolysis (69). This strategy 


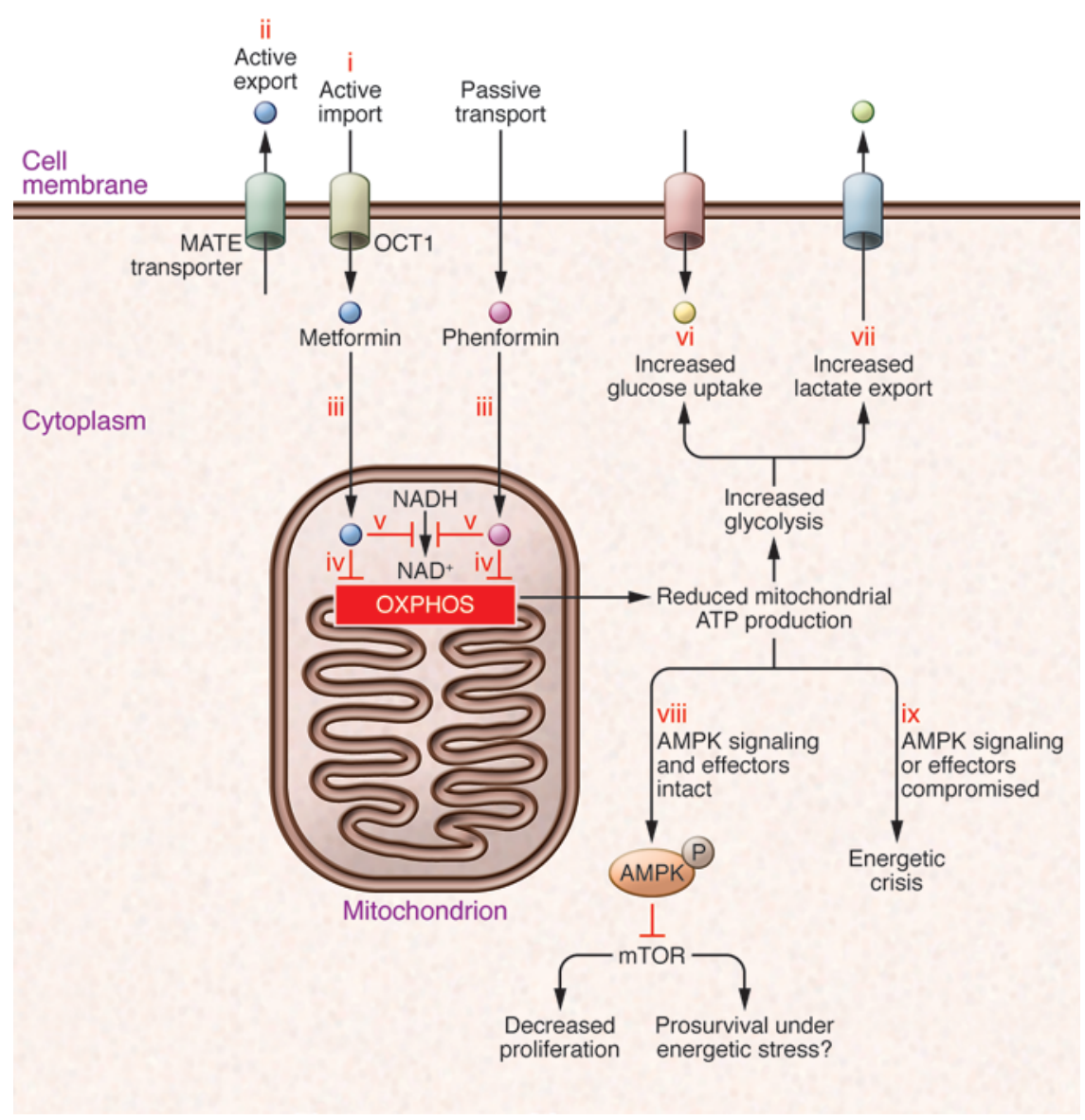

\section{Figure 2}

Cellular effects of biguanides. (i) Uptake of some biguanides, such as metformin, is dependent on specific transport molecules such as OCT1; other biguanides, such as phenformin, are more lipophilic and less dependent on active transport. (ii) Biguanides can be actively excreted, for example by multidrug and toxin extrusion (MATE) proteins. (iii) Biguanides are not homogeneously distributed within the cell; the mitochondrial membrane potential promotes increased uptake of biguanides, and mitochondria have the highest concentration. This is important, as key targets such as respiratory complex I, are in the mitochondria. Mitochondrial actions of biguanides reduce oxidative phosphorylation, resulting in decreased cellular ATP (iv), decreased NAD+ (v), and other derangements in mitochondrial metabolism. The reduction in ATP generation from oxidative phosphorylation leads to a compensatory increase in glucose uptake (vi) and glycolysis, with increased lactate secretion (vii). However, especially if glucose concentrations are limiting, this compensation is not sufficient to restore ATP to baseline levels. (viii) Therefore, if AMPK and its effectors are functional, AMPK activation results in reductions in energy expenditure and anabolic processes, leading to an antiproliferative (but potentially prosurvival) effect. (ix) On the other hand, in cancers with loss of function of AMPK or its key effectors, energy expenditure is not reduced despite reduced energy supply, leading to an energetic crisis. would serve to block the compensatory increase in glycolytic ATP production that is seen when oxidative phosphorylation is reduced by biguanides. While this approach does indeed result in enhanced cytotoxicity, it may not be practical for clinical application unless drugs are developed that safely and effectively inhibit glycolysis.

DNA-damaging agents. Another intriguing possibility, supported by relatively few models, is that biguanides may enhance activity of DNA-damaging agents such as ionizing radiation or cis platinum. A hypothesis in this context relates to the energetic cost of DNA repair. While normal rates of DNA repair have little influence on cellular energy homeostasis, the massive and sudden DNA damage following exposure to certain cytotoxic agents leads to abnormally high levels of PARP-mediated ribosylation, which transiently involves energy consumption high enough to deplete ATP levels, at least in certain models $(70,71)$. This finding raises the somewhat radical concept that a portion of the therapeutic benefit of cytotoxic agents may result not from DNA damage, as is generally supposed, but rather from a transient energetic stress secondary to abnormally high rate of DNA repair following DNA damage. If this hypothesis is valid, then there may be a strategic role for combining biguanides, perhaps at high doses but for a limited duration, at a specific time following administration of DNA-damaging agents. This combination might intensify energetic stress in cancer cells by limiting ATP production at the time of increased utilization. While this might enhance toxicity in normal tissues as well, there is the possibility of a favorable therapeutic index in cases in which genetic lesions in cancer cells, such as loss of func- tion of LKB1, result in enhanced proliferation but increased vulnerability to energetic stress.

Anti-VEGF therapies. An adaptive response of a cancer cell to energetic stress caused by a biguanide is to encourage the delivery of additional carbon sources and oxygen by stimulating new vessel formation. Increased VEGF expression is known to occur following AMPK activation, so it is not surprising that some tumors secrete this factor on exposure to biguanides. This process has been associated with metformin-induced acceleration of tumor growth (72), a finding which should raise a cautionary note. On the other hand, this model documented that co-administration of a VEGF inhibitor with a biguanide resulted in antineoplastic activity greater than that seen with either agent alone.

Oncogenic kinase inhibitors. Several contexts for combination therapy with kinase inhibitors are of interest. The systemic effect of metformin in reducing the hyperinsulinemia of insulin resistance suggests a potential role in combination with AKT or PI3K inhibitors, as hyperglycemia and hyperinsulinemia are known metabolic consequences of these agents that cause toxicity and limit treatment efficacy in laboratory models (M.-J. Blouin, personal communication).

In the case of inhibition of the serine/threonine-protein kinase Braf in the treatment of melanoma $(50,73,74)$, experimental evidence indicates that kinase inhibition results in an acute reduction of glycolysis, which results either in cell death or a PGC1 $\alpha$-mediated switch to oxidative phosphorylation, in a sense a "reverse Warburg effect" $(73,74)$. While further oncogenic mutations may revert cells to a Warburg phenotype, the possibility that blocking the 
acute compensatory increase in oxidative phosphorylation would enhance efficacy of the kinase inhibition deserves examination (50). $m$ TOR inhibitors. Biguanide-induced energy stress may inhibit mTOR via AMPK (75). From this point of view, it would seem redundant for pharmacologic therapies to directly target mTOR in addition to AMPK. However, this combination deserves study because mTOR inhibition alone leads to the inhibition of mRNA translation and other energy-consuming processes, which improve cellular energy balance. Thus, the combination may be regarded as adding energetic stress to $\mathrm{mTOR}$ inhibition, rather than targeting $\mathrm{mTOR}$ in a redundant fashion.

Salicylates. A recent report (76) demonstrated that salicylate can directly interact with and activate AMPK at concentrations that do not result in impairment of mitochondrial function or ATP production. In therapeutic settings such as adjuvant treatment or cancer risk reduction, in which the impact of long-term AMPK activation is to be studied, it is possible that a strategy of co-administration of moderate doses of salicylate and metformin would be better tolerated than a high dose of either agent alone.

Blockers of lactate $/ \mathrm{H}^{+}$transport. Most cells, when exposed to sufficient biguanide concentration, attempt to cope with the resulting energetic stress by increasing glycolysis, which leads to increased lactic acid secretion. In an in vitro model system, impressive synthetic lethality resulted when cells were simultaneously exposed to a biguanide and agents that interfere with lactate $/ \mathrm{H}^{+}$transport (J. Pouyssegur, personal communication). This cell death was a consequence of the ability of biguanides to inhibit oxidative phosphorylation and reduce TCA cycle flux, creating a requirement for maximal glycolysis while simultaneously using another agent to limit excretion of the glycolytic end product. This combination is now being evaluated in vivo (77).

Androgen-targeting therapies in prostate cancer. Androgen deprivation is a mainstay of prostate cancer treatment, but is associated with hyperinsulinemia, which may contribute to both long-term toxicity and treatment failure $(33,78)$. If the hyperinsulinemia associated with androgen deprivation proves to be as responsive to metformin as the hyperinsulinemia of type II diabetes, it is plausible that combined androgen deprivation and metformin would be both better tolerated and more effective than androgen deprivation alone.

\section{Pharmacokinetics and dosing}

The actions of biguanides in model systems will have clinical relevance only if the drug accumulates to a sufficient extent in target tissues in patients. Conventional antidiabetic doses of metformin are known to be sufficient to result in inhibition of hepatic gluconeogenesis, but liver may not be representative of other tissues of potential interest. This organ accumulates relatively high metformin concentrations not only because it receives the portal circulation, which provides higher concentrations than the systemic circulation following oral dosing, but also because hepatocytes express high levels of OCT1, which enhances cellular uptake of the drug $(24,25,79)$. There is also evidence for a high concentration of metformin in the gut following oral exposure (80), so from a pharmacokinetic perspective, liver and colon are particularly interesting sites for potential clinical applications.

For applications in which long-term administration is contemplated, it is unlikely that metformin can be given orally at doses higher than 1,500-2,000 mg/day. However, in contexts in which short-term exposure is planned, higher doses of metformin or other biguanides may be feasible. While all studies that have com- pared metformin with phenformin have documented higher antineoplastic activity of the latter $(26,81,82)$, it is important to note that formal preclinical studies comparing antineoplastic activity of metformin, phenformin, or other biguanides, each administered at maximal tolerated dose, have not yet been reported.

Drug delivery may be optimized by careful selection of existing or novel biguanides, by liposomal formulations, or even by methods to enhance delivery to desired cell populations by antibodymediated targeting strategies. Little research has been undertaken in these areas to date: justification for such efforts would be clear if biguanides are shown to have significant activity in model systems at doses that are currently impractical to administer to patients. This scenario might increase the interest of the pharmaceutical industry, as novel formulations or derivatives of existing biguanides may create opportunities to protect intellectual property more easily than is the case for metformin, which of course is a very widely used generic drug.

\section{Clinical contexts for trials and patient selection}

Preclinical research on biguanides carried out to date provides little guidance for prioritization of specific oncologic indications for study. Pharmacokinetic considerations suggest that liver and colon deserve attention, but epidemiologic and laboratory studies raise the possibility of activity for many cancers. Apart from the large number of different cancers under study, there are several clinical contexts for each of these, including prevention, adjuvant treatment, and palliative treatment, and in each case, treatment involves either single-agent therapy or a variety of drug combinations. This situation, together with the wide availability and low cost of metformin, also contributes to the large number of trials in progress. Thus, the manner in which metformin is being studied clinically differs from the conventional scenario in which a novel agent is subjected to phase II evaluation for specific indications suggested by preclinical studies.

Most trials underway are exploring broad indications for common cancers. However, certain specific indications for risk reduction or treatment deserve special consideration. These include risk reduction in polyposis syndromes including Peutz-Jaegers and in Li-Fraumeni syndrome (61). For palliative treatment, perhaps in strategic drug combinations, trials for tumors with molecular characteristics predictive of defects in tolerance to energetic stress, such as loss of function of LKB1, may deserve prioritization. It is also possible that activity related to indirect mechanisms will be confined to those patients with significant baseline hyperinsulinemia.

\section{Resistance mechanisms}

Several potential resistance mechanisms for biguanides in oncology have been proposed. If metformin-induced reduction of insulin level is of a sufficient magnitude to reduce proliferation of the subset of tumors that are insulin dependent, one might anticipate selection for activating mutations of PI3K that would confer autonomy from insulin, thus reducing benefit. Recent models indicate that the resistance to direct actions of biguanides can emerge at early time points after treatment (56). The mechanisms are not well defined but could include selection for processes that prevent cellular drug accumulation or processes that increase mitochondrial number, to compensate for the biguanide-induced reduction in ATP output per mitochondrion. Another possibility to consider is that activation of AMPK, while antiproliferative, may in certain contexts also be prosurvival, as energetic requirements are reduced (83). Resistance devel- 
ops to virtually all antineoplastic therapies, so these considerations on their own should not discourage further research. Rather, a better understanding of the mechanisms of resistance may suggest additional rational drug combinations and/or patient selection criteria.

\section{Early clinical results}

More than 100 clinical trials of metformin in cancer treatment are listed as active on the NIH ClinicalTrials.gov web site (84). These trials are evaluating the specific hypothesis that the antidiabetic dose of metformin has antineoplastic activity. While this is clearly an attractive possibility, it is not directly supported by preclinical studies, which generally demonstrate activity at higher concentrations than are seen in the serum of metformin-treated diabetics. To date, only metformin is being studied clinically, despite clues from laboratory studies that phenformin may have greater antineoplastic activity $(26,81,82,85)$. For all of these reasons, negative results of these early trials should not be used to justify ceasing research on effects of biguanides on cancer. On the other hand, if antineoplastic activity is detected, it is likely that further optimization will be possible.

Presently available clinical trial results involve intermediate or surrogate outcome measurements, such as changes in cellular proliferation or hormone levels, rather than direct measures of clinical benefit. While studies of many disease sites are in progress, most data available at this time deal with colon or breast cancer.

An early study (86) examined short-term effects of metformin on breast cancer cell proliferation in nondiabetic women with operable breast cancer by comparing serum and tissue biomarkers obtained at baseline and following drug administration. This study was not placebo controlled and did not examine postprandial insulin or glucose levels. Metformin did not alter fasting insulin levels, but a small decline in tumor cell proliferation, as estimated by Ki-67 staining, was observed. Another larger $(N=200)$ study $(87)$ of similar design was carried out in a randomized, placebo-controlled manner - an obvious strength. However, the tissue procurement method was not identical for the baseline and follow-up samples, and there was variability in the time between the last metformin dose and the point at which the surgical specimen was obtained. These issues complicate interpretation of the findings, including the fact that Ki-67-estimated proliferation rates increased between biopsy and surgery in placebo-treated women. This puzzling observation has been observed in other studies of similar design that did not involve metformin (88, 89). This poorly understood rise was attenuated in women receiving metformin, particularly in subsets characterized by high BMI or high levels of C-peptide or insulin-like binding protein 1. A third study of similar design (90) was smaller $(N=39)$, not placebo controlled, and compared tissue from needle biopsies with surgical specimens. Nonsignificant declines in insulin levels together with increased apoptosis and reduced proliferation were observed. Available data suggest that the interpersonal variability in baseline fasting insulin level is larger in magnitude than the metformin-induced declines, so it remains to be seen whether metformin exposure is indeed a major determinant of insulin levels in individuals without diabetes. An earlier trial that used a somatostatin analog to lower insulin and IGF-I levels in breast cancer patients achieved small-magnitude reductions in the hypothesized direction without an effect on breast cancer outcomes (29), but this result cannot be used to rule out an effect of small-magnitude declines, as the duration of therapy was unexpectedly limited by gastrointestinal toxicity.

Taken together, these early biomarker trials do not allow definitive conclusions. Certainly the observed metformin-induced changes in proliferation are small compared with those seen with approved drugs such as aromatase inhibitors (91). The studies that included untreated controls raise the possibility of a perioperative elevation of insulin, perhaps related to perioperative intravenous glucose infusion, that raises proliferation and is attenuated by metformin.

With respect to the colon, available data deal with the effects of metformin (in nondiabetic subjects) on normal epithelial cells rather than cancer cells and are therefore more relevant to hypotheses concerning the use of metformin for prevention than for treatment. Low-dose $(250 \mathrm{mg} / \mathrm{d})$ oral metformin reduced the number of aberrant crypt foci (a premalignant change) in a short-term trial (92). Although the effect of metformin at a dose lower than that conventionally used in diabetes treatment on insulin levels was not studied, this would not be expected. Another trial involving baseline and onmetformin colon mucosa biopsies demonstrated that conventional antidiabetic doses of metformin reduce epithelial proliferation and have a larger effect on postprandial than fasting insulin levels, but did not establish the mechanism underlying the antiproliferative effect (93). A direct effect attributable to high luminal metformin concentrations (80) is plausible. Increased intestinal glucose uptake in patients receiving metformin have been observed using F-18 fluoro-2-deoxyglucose PET (FDG-PET) (94). This observation may represent a pharmacodynamic marker of compensatory increase in glucose uptake arising in the epithelial cells as a consequence of metformin-induced reduction in oxidative phosphorylation (95).

\section{Concluding perspectives}

Many compounds are being evaluated as metabolic therapies for cancer. Metformin deserves to be included in this group, but it is occasionally omitted because it represents a case of drug repurposing rather than novel drug development. It is clear that metformin is now being studied in clinical trials more intensively than other proposed metabolic therapies. This is attributable to its widespread availability and safety and to attractive mechanistic hypotheses, even though gaps in knowledge regarding mode of action and pharmacokinetics represent challenges to optimum trial design.

While the original rationales for studying metformin activity for cancer - the lowering of insulin levels systemically and the activation of AMPK in tumors - are still of interest, these are now recognized to represent only the tip of the iceberg in terms of potential mechanisms. A large number of ongoing clinical trials are studying metformin for indications in oncology, but all are limited to the specific hypothesis that antidiabetic doses of metformin have antineoplastic activity. A finding of clinical utility in these trials would represent an important achievement of repurposing research. Of particular significance would be the fact that metformin is well tolerated and inexpensive: the introduction of a generic drug for diabetes as a novel antineoplastic agent would be an unusual and happy case history in drug development.

Some possibilities that are not being evaluated by ongoing trials may deserve future clinical study, depending on results of ongoing preclinical work. These include evaluation of biguanides other than metformin with optimized pharmacokinetic characteristics, the short-term use of metformin at higher doses than are commonly used in diabetes, the use of biguanides in combination with other agents that may lead to synthetic lethality, and the possibility of specifically targeting cancers that have defects in homeostatic systems that allow survival under energetic stress. If the initial clinical trials show activity, these areas of investigation will deserve further attention. But even if results from the first generation of 
clinical trials are disappointing, these topics should be investigated further to ensure that all contexts in which biguanides might be useful are fully explored.

\section{Acknowledgments}

The author acknowledges helpful discussions with Judy Hirst (Cambridge University) and Nahum Sonenberg and Ivan Topise- rovic (McGill University) and apologizes to colleagues whose work could not be cited due to space limitations.

Address correspondence to: Michael Pollak, Department of Oncology, McGill University, Montreal, Quebec, Canada H3W 2B7. Phone: 514.340.8222, ext. 4139; Fax: 514.340.8600; E-mail: michael.pollak@mcgill.ca.
1. Koppenol WH, Bounds PL, Dang CV. Otto Warburg's contributions to current concepts of cancer metabolism. Nat Rev Cancer. 2011;11(5):325-337.

2. Pollak MN. Investigating metformin for cancer prevention and treatment: the end of the beginning. Cancer Discov. 2012;2(9):778-790.

3. Turner N, et al. Berberine and its more biologically available derivative, dihydroberberine, inhibit mitochondrial respiratory complex I: a mechanism for the action of berberine to activate AMP-activated protein kinase and improve insulin action. Diabetes. 2008;57(5):1414-1418.

4. Falcone AB, Mao RL, Shrago E. A study of the action of hypoglycemia-producing biguanide and sulfonylurea compounds on oxidative phosphorylation. J Biol Chem. 1962;237:904-909.

5. Ota S, et al. Metformin suppresses glucose-6-phosphatase expression by a complex I inhibition and AMPK activation-independent mechanism. Biochem Biophys Res Commun. 2009;388(2):311-316.

6. Owen MR, Doran E, Halestrap AP. Evidence that metformin exerts its anti-diabetic effects through inhibition of complex 1 of the mitochondrial respiratory chain. Biochem J. 2000;348(pt 3):607-614.

7. El-Mir MY, Nogueira V, Fontaine E, Avéret N, Rigoulet M, Leverve X. Dimethylbiguanide inhibits cell respiration via an indirect effect targeted on the respiratory chain complex I. J Biol Chem. 2000; 275(1):223-228.

8. Ouyang J, Parakhia RA, Ochs RS. Metformin activates AMP kinase through inhibition of AMP deaminase. J Biol Chem. 2011;286(1):1-11.

9. Logie L, et al. Cellular responses to the metalbinding properties of metformin. Diabetes. 2012 61(6):1423-1433.

10. Nestler JE. Metformin for the treatment of the polycystic ovary syndrome. $N$ Engl J Med. 2008; 358(1):47-54.

11. Chen Ch, et al. Effects of adjunctive metformin on metabolic traits in nondiabetic clozapine-treated patients with schizophrenia and the effect of metformin discontinuation on body weight: a 24-week, randomized, double-blind, placebo-controlled study. J Clin Psychiatry. 2013;74(5):e424-e330.

12. Evans JM, Donnelly LA, Emslie-Smith AM, Alessi DR, Morris AD. Metformin reduced risk of cancer in diabetic patients. BMJ. 2005;330(7503):1304-1305.

13. Zhang P, Li H, Tan X, Chen L, Wang S. Association of metformin use with cancer incidence and mortality: A meta-analysis. Cancer Epidemiol. 2013; 37(3):207-218

14. Noto H, Goto A, Tsujimoto T, Noda M. Cancer risk in diabetic patients treated with metformin: a systematic review and meta-analysis. PLoS One. 2012; 7(3):e33411.

15. Suissa S. Immortal time bias in pharmaco-epidemiology. Am J Epidemiol. 2008;167(4):492-499.

16. Suissa S, Azoulay L. Metformin and the risk of cancer: time-related biases in observational studies. Diabetes Care. 2012;35(12):2665-2673.

17. Nkontchou $G$, et al. Impact of metformin on the prognosis of cirrhosis induced by viral hepatitis C in diabetic patients. J Clin Endocrinol Metab. 2011;96(8):2601-2608

18. Lee MS, Hsu CC, Wahlqvist ML, Tsai HN, Chang YH, Huang YC. Type 2 diabetes increases and metformin reduces total, colorectal, liver and pancreatic cancer incidences in Taiwanese: a representative population prospective cohort study of 800,000 individuals. BMC Cancer. 2011;11:20

19. Bodmer M, Meier C, Krähenbühl S, Jick SS, Meier CR. Long-term metformin use is associated with decreased risk of breast cancer. Diabetes Care. 2010; 33(6):1304-1308.

20. Lega IC, Austin PC, Gruneir A, Goodwin PJ, Rochon PA, Lipscombe LL. Association between metformin therapy and mortality after breast cancer: a population-based study [published online ahead of print April 30, 2013]. Diabetes Care. doi:10.2337/dc12-2535.

21. Zakikhani M, Dowling R, Fantus IG, Sonenberg N, Pollak M. Metformin is an AMP kinase-dependent growth inhibitor for breast cancer cells. Cancer Res. 2006;66(21):10269-10273.

22. Anisimov VN, et al. Effect of metformin on life span and on the development of spontaneous mammary tumors in HER-2/neu transgenic mice. Exp Gerontol. 2005;40(8-9):685-693.

23. Dykens JA, Jamieson J, Marroquin L, Nadanaciva $\mathrm{S}$, Billis PA, Will Y. Biguanide-induced mitochondrial dysfunction yields increased lactate production and cytotoxicity of aerobically-poised HepG2 cells and human hepatocytes in vitro. Toxicol Appl Pharmacol. 2008;233(2):203-210.

24. Gong L, Goswami S, Giacomini KM, Altman RB, Klein TE. Metformin pathways: pharmacokinetics and pharmacodynamics. Pharmacogenet Genomics. 2012;22(11):820-827.

25. Nies AT, Koepsell H, Damme K, Schwab M. Organic cation transporters (OCTs, MATEs), in vitro and in vivo evidence for the importance in drug therapy. Handb Exp Pharmacol. 2011;(201):105-167.

26. Segal ED, Yasmeen A, Beauchamp MC, Rosenblatt J, Pollak M, Gotlieb WH. Relevance of the OCT1 transporter to the antineoplastic effect of biguanides. Biochem Biophys Res Commun. 2011;414(4):694-699.

27. Shaw RJ, et al. The kinase LKB1 mediates glucose homeostasis in liver therapeutic effects of metformin. Science. 2005;310(5754):1642-1646.

28. Foretz M, et al. Metformin inhibits hepatic gluconeogenesis in mice independently of the LKB1/ AMPK pathway via a decrease in hepatic energy state. J Clin Invest. 2010;120(7):2355-2369.

29. Pritchard KI, et al. Randomized trial of tamoxifen versus combined tamoxifen and octreotide LAR Therapy in the adjuvant treatment of early-stage breast cancer in postmenopausal women: NCIC CTG MA.14. JClin Oncol. 2011;29(29):3869-3876.

30. Goodwin PJ, et al. Insulin- and obesity-related variables in early-stage breast cancer: correlations and time course of prognostic associations. J Clin Oncol. 2012;30(2):164-171.

31. Ma J, et al. A prospective study of plasma C-peptide and colorectal cancer risk in men.J Natl Cancer Inst. 2004;96(7):546-553.

32. Wolpin BM, et al. Insulin, the insulin-like growth factor axis, and mortality in patients with nonmetastatic colorectal cancer. J Clin Oncol. 2009;27(2):176-185.

33. Ma J, et al. Prediagnostic body-mass index, plasma C-peptide concentration, and prostate cancer-specific mortality in men with prostate cancer: a long-term survival analysis. Lancet Oncol. 2008;9(11):1039-1047.

34. Venkateswaran V, et al. Association of diet-induced hyperinsulinemia with accelerated growth of prostate cancer (LNCaP) xenografts. J Natl Cancer Inst. 2007;99(23):1793-1800
35. Ferguson RD, et al. Hyperinsulinemia enhances c-Myc-mediated mammary tumor development and advances metastatic progression to the lung in a mouse model of type 2 diabetes. Breast Cancer Res. 2012;14(1):R8.

36. Algire C, Amrein L, Bazile M, David S, Zakikhani M, Pollak M. Diet tumor LKB1 expression interact to determine sensitivity to anti-neoplastic effects of metformin in vivo. Oncogene. 2011;30(10):1174-1182.

37. Pollak M. The insulin and insulin-like growth factor receptor family in neoplasia: an update. Nat Rev Cancer. 2012;12(3):159-169.

38. Hvid H, et al. Stimulation of MC38 tumor growth by insulin analog X10 involves the serine synthesis pathway. Endocr Relat Cancer. 2012;19(4):557-574.

39. Pearce EL, et al. Enhancing CD8 T-cell memory by modulating fatty acid metabolism. Nature. 2009;460(7251):103-107.

40. Moiseeva O, et al. Metformin inhibits the senescence-associated secretory phenotype by interfering with IKK/NF-KB activation. Aging Cell. 2013; 12(3):489-498

41. Hirsch HA, Iliopoulos D, Struhl K. Metformin inhibits the inflammatory response associated with cellular transformation and cancer stem cell growth. Proc Natl Acad Sci U S A. 2013;110(3):972-977.

42. Evia-Viscarra ML, et al. The effects of metformin on inflammatory mediators in obese adolescents with insulin resistance: controlled randomized clinical trial. J Pediatr Endocrinol Metab. 2012;25(1-2):41-49.

43. Gómez-Díaz RA, et al. Metformin decreases plasma resistin concentrations in pediatric patients with impaired glucose tolerance: a placebo-controlled randomized clinical trial. Metabolism. 2012; 61(9):1247-1255.

44. Miller RA, Chu Q, Xie J, Foretz M, Viollet B, Birnbaum MJ. Biguanides suppress hepatic glucagon signalling by decreasing production of cyclic AMP. Nature. 2013;494(7436):256-260.

45. Singh S, Akhtar N, Ahmad J. Plasma adiponectin levels in women with polycystic ovary syndrome: impact of metformin treatment in a case-control study. Diabetes Metab Syndr. 2012;6(4):207-211.

46. Cabreiro F, et al. Metformin retards aging in C. elegans by altering microbial folate and methionine metabolism. Cell. 2013;153(1):228-239.

47. Corominas-Faja B, et al. Metabolomic fingerprint reveals that metformin impairs one-carbon metabolism in a manner similar to the antifolate class of chemotherapy drugs. Aging (Albany NY). 2012; 4(7):480-498

48. Schulze A, Harris AL. How cancer metabolism is tuned for proliferation and vulnerable to disruption. Nature. 2012;491(7424):364-373.

49. Wallace DC. Mitochondria and cancer. Nat Rev Cancer. 2012;12(10):685-698.

50. Pollak M. Targeting oxidative phosphorylation: why, when, and how. Cancer Cell. 2013;23(3):263-264.

51. Hardie DG. The LKB1-AMPK pathway-friend or foe in cancer? Cancer Cell. 2013;23(2):131-132.

52. Hardie DG, Ross FA, Hawley SA. AMPK: a nutrient and energy sensor that maintains energy homeostasis. Nat Rev Mol Cell Biol. 2012;13(4):251-262.

53. Algire C, Amrein L, Zakikhani M, Panasci L, Pollak M. Metformin blocks the stimulative effect of a high energy diet on colon carcinoma growth in vivo and is associated with reduced expression of fatty acid acid synthase. Endocr Relat Cancer. 2010;17(2):351-360. 
54. Larsson O, et al. Distinct perturbation of the translatome by the antidiabetic drug metformin. Proc Natl Acad Sci U S A. 2012;109(23):8977-8982.

55. Javeshghani $\mathrm{S}$, et al. Carbon source myc expression influence the antiproliferative actions of metformin. Cancer Res. 2012;72(23):6257-6267.

56. Shackelford DB, et al. LKB1 inactivation dictates therapeutic response of non-small cell lung cancer to the metabolism drug phenformin. Cancer Cell. 2013;23(2):143-158

57. Jones RG, Thompson CB. Tumor suppressors and cell metabolism: a recipe for cancer growth. Genes Dev. 2009;23(5):537-548.

58. Hadad SM, et al. Histological evaluation of AMPK signalling in primary breast cancer. BMC Cancer. 2009;9:307.

59. Sanchez-Cespedes M, et al. Inactivation of LKB1/ STK11 is a common event in adenocarcinomas of the lung. Cancer Res. 2002;62(13):3659-3662.

60. Duivenvoorden WC, et al. Underexpression of tumour suppressor LKB1 in clear cell renal cell carcinoma is common and confers growth advantage in vitro and in vivo. Br J Cancer. 2013;108(2):327-333.

61. Wang PY, et al. Increased oxidative metabolism in the Li-Fraumeni syndrome. N Engl J Med. 2013; 368(11):1027-1032.

62. Matoba S, et al. p53 regulates mitochondrial respiration. Science. 2006;312(5780):1650-1653.

63. Jones RG, et al. AMP-activated protein kinase induces a p53-dependent metabolic checkpoint. Mol Cell. 2005;18(3):283-293.

64. Gou S, Cui P, Li X, Shi P, Liu T, Wang C. Low concentrations of metformin selectively inhibit $\mathrm{CD}_{133^{+}}$cell proliferation in pancreatic cancer and have anticancer action. PLoS One. 2013;8(5):e63969.

65. Hirsch HA, Iliopoulos D, Tsichlis PN, Struhl K Metformin selectively targets cancer stem cells acts together with chemotherapy to block tumor growth prolong remission. Cancer Res. 2009 69(19):7507-7511.

66. Kalender A, et al. Metformin, independent of AMPK inhibits $\mathrm{mTORC} 1$ in a rag GTPase-dependent manner. Cell Metab. 2010;11(5):390-401.

67. Zoncu R, Bar-Peled L, Efeyan A, Wang S, Sancak Y, Sabatini DM. mTORC1 senses lysosomal amino acids through an inside-out mechanism that requires the vacuolar $\mathrm{H}(+)$-ATPase. Science. 2011; 334(6056):678-683.

68. Ben Sahra I, et al. Metformin, independent of AMPK, induces mTOR inhibition and cell-cycle arrest through REDD1. Cancer Res. 2011;71(13):4366-4372.
69. Ben Sahra I, et al. Targeting cancer cell metabolism: the combination of metformin and 2-deoxyglucose induces p53-dependent apoptosis in prostate cancer cells. Cancer Res. 2010;70(6):2465-2475.

70. Zong WX, Ditsworth D, Bauer DE, Wang ZQ, Thompson CB. Alkylating DNA damage stimulates a regulated form of necrotic cell death. Genes Dev. 2004;18(11):1272-1282.

71. Affar el B, Shah RG, Dallaire AK, Castonguay V, Shah GM. Role of poly(ADP-ribose) polymerase in rapid intracellular acidification induced by alkylating DNA damage. Proc Natl Acad Sci U S A. 2002; 99(1):245-250

72. Martin MJ, Hayward R, Viros A, Marais R. Metformin accelerates the growth of BRAF V600E-driven melanoma by upregulating VEGF-A. Cancer Discov. 2012;2(4):344-355.

73. Haq R, et al. Oncogenic BRAF regulates oxidative metabolism via PGC1 $\alpha$ and MITF. Cancer Cell. 2013;23(3):302-315.

74. Vazquez F, et al. PGC1 $\alpha$ expression defines a subset of human melanoma tumors with increased mitochondrial capacity and resistance to oxidative stress. Cancer Cell. 2013;23(3):287-301.

75. Gwinn DM, et al. AMPK phosphorylation of raptor mediates a metabolic checkpoint. Mol Cell. 2008;30(2):214-226.

76. Hawley SA, et al. The ancient drug salicylate directly activates AMP-activated protein kinase. Science. 2012;336(6083):918-922.

77. Le Floch R, et al. CD147 subunit of lactate/H+ symporters MCT1 hypoxia-inducible MCT4 is critical for energetics growth of glycolytic tumors. Proc Natl Acad Sci U S A. 2011;108(40):16663-16668.

78. Lubik AA, et al. Insulin directly increases de novo steroidogenesis in prostate cancer cells. Endocr Relat Cancer. 2013;20(2):173-186.

79. Quaile MP, et al. Toxicity and toxicokinetics of metformin in rats. Toxicol Appl Pharmacol. 2010; 243(3):340-347.

80. Vidon N, Chaussade S, Noel M, Franchisseur C, Huchet B, Bernier JJ. Metformin in the digestive tract. Diabetes Res Clin Pract. 1988;4(3):223-229.

81. Appleyard MV, et al. Phenformin as prophylaxis therapy in breast cancer xenografts. BrJ Cancer. 2012; 106(6):1117-1122.

82. Zhu Z, Jiang W, McGinley J, Thompson H. Comparison of of metformin, phenformin and buformin in mammary carcinogenesis in nonobese, non diabetic rats. In: Proceedings of the 104th Annual Meeting of the American Association for Cancer Research;
April 6-10, 2013; Washington, DC. Abstract 2272.

83. Jeon SM, Chandel NS, Hay N. AMPK regulates NADPH homeostasis to promote tumour cell survival during energy stress. Nature. 2012; 485(7400):661-665.

84. NIH. ClinicalTrials.gov. NIH Web site. Accessed July 23, 2013.

85. Huang $X$, et al. Important role of the LKB1-AMPK pathway in suppressing tumourigenesis in PTEN deficient mice. Biochem J. 2008;412(2):211-221.

86. Hadad S, et al. Evidence for biological effects of metformin in operable breast cancer: a pre-operative, window-of-opportunity, randomized trial. Breast Cancer Res Treat. 2011;128(3):783-794.

87. Bonanni B, et al. Dual effect of metformin on breast cancer proliferation in a randomized presurgical trial. J Clin Oncol. 2012;30(21):2593-2600.

88. Decensi A, et al. Lapatinib activity in premalignant lesions HER-2-positive cancer of the breast in a randomized, placebo-controlled presurgical trial. Cancer Prev Res (Phila). 2011;4(8):1181-1189.

89. Decensi A, et al. A randomized trial of low-dose tamoxifen on breast cancer proliferation and blood estrogenic biomarkers. J Natl Cancer Inst. 2003; 95(11):779-790.

90. Niraula S, et al. Metformin in early breast cancer: A prospective, open-label, neoadjuvant window of opportunity study. Breast Cancer Res Treat. 2012; 135(3):821-830.

91. Ellis MJ, et al. Randomized phase II neoadjuvant comparison between letrozole, anastrozole, and exemestane for postmenopausal women with estrogen receptor-rich stage 2 to 3 breast cancer: clinical and biomarker outcomes and predictive value of the baseline PAM50-based intrinsic subtype - ACOSOG Z1031. JClin Oncol. 2011;29(17):2342-2349.

92. Hosono K, et al. Metformin suppresses colorectal aberrant crypt foci in a short-term clinical trial. Cancer Prev Res (Phila). 2010;3(9):1077-1083.

93. Blouin M, et al. Metformin and biomarkers relevant to neoplasia in nondiabetic patients [abstract e 14615]. J Clin Oncol. 2013;31(suppl).

94. Gontier E, et al. High and typical 18F-FDG bowel uptake in patients treated with metformin. Eur J Nucl Med Mol Imaging. 2008;35(1):95-99.

95. Habibollahi P, van den Berg NS, Kuruppu D, Loda M, Mahmood U. Metformin - an adjunct antineoplastic therapy - divergently modulates tumor metabolism and proliferation, interfering with early response prediction by $18 \mathrm{~F}-\mathrm{FDG}$ PET imaging. J Nucl Med. 2013;54(2):252-258. 